\title{
Twitter earthquake detection: earthquake monitoring in a social world
}

\author{
Paul S. Earle ${ }^{\star}$, Daniel C. Bowden, Michelle Guy
}

U.S. Geological Survey, Denver, CO, USA

\author{
Article history \\ Received August 10, 2011; accepted October 14, 2011. \\ Subject classification: \\ Surveys, measurements and monitoring, Instruments and techniques, Data processing, Seismological data, General or miscellaneous.
}

\section{ABSTRACT}

The U.S. Geological Survey (USGS) is investigating how the social networking site Twitter, a popular service for sending and receiving short, public text messages, can augment USGS earthquake response products and the delivery of hazard information. Rapid detection and qualitative assessment of shaking events are possible because people begin sending public Twitter messages (tweets) with in tens of seconds after feeling shaking. Here we present and evaluate an earthquake detection procedure that relies solely on Twitter data. A tweet-frequency time series constructed from tweets containing the word "earthquake" clearly shows large peaks correlated with the origin times of widely felt events. To identify possible earthquakes, we use a short-term-average, long-term-average algorithm. When tuned to a moderate sensitivity, the detector finds 48 globallydistributed earthquakes with only two false triggers in five months of data. The number of detections is small compared to the 5,175 earthquakes in the USGS global earthquake catalog for the same five-month time period, and no accurate location or magnitude can be assigned based on tweet data alone. However, Twitter earthquake detections are not without merit. The detections are generally caused by widely felt events that are of more immediate interest than those with no human impact. The detections are also fast; about $75 \%$ occur within two minutes of the origin time. This is considerably faster than seismographic detections in poorly instrumented regions of the world. The tweets triggering the detections also provided very short first-impression narratives from people who experienced the shaking.

\section{Introduction}

Twitter is a service that allows anyone to send and receive 140-character messages (tweets) via text message and Internetenabled devices. Tweets can be sent and received through a webpage, a mobile device, or third-party Twitter applications. Tweets can be sent publicly or privately to a specified user. All users who opt to 'follow' a Twitter user will receive that user's public tweets. It is these public messages that really separate Twitter from instant message services that typically involve one-to-one communication rather than one-to-many. In addition to receiving tweets from users you elect to follow, Twitter provides public access to its database. One can freely search and download recent tweets containing any keywords of interest, thus providing real-time access to Twitter discussions on any topic anywhere around the globe.

Within seconds of widely felt earthquakes around the world, narratives and exclamations of 140-character or less are publicly distributed on Twitter. These tweets sometimes number in the thousands and some provide a qualitative description of the shaking effects. These short accounts of shaking effects are sometimes available before the seismically derived estimates of location and magnitude are publicly distributed by the U.S. Geological Survey (USGS) [e.g., Earle et al. 2010]. Since this information is easily accessible and rapidly available, people are increasingly turning to Twitter and other forms of social media to gather rapid situational awareness following earthquakes and other disasters [e.g., Liu and Ayala Iacucci 2010, Mendoza et al. 2010, Palen et al. 2010a]. As a distributor of authoritative earthquake information, the USGS is investigating the potential benefits and pitfalls of using Twitter to augment its suite of seismically derived earthquake products and alerts. Possible uses fall into the basic categories of alert dissemination, situational awareness, and event detection.

\section{Alert dissemination}

Earthquake alert dissemination is a straightforward use of Twitter. Broadcasting earthquake alerts via Twitter has several advantages. It provides an additional method to reach large segments of the population who are becoming more reliant on social media as a means of communication and information gathering. Twitter is fast; Twitter messages are generally available to all followers within seconds of being submitted. Users leverage Twitter's delivery infrastructure such that once a message delivery is set up there is no additional overhead for the user to reach more people. In several countries, Twitter also allows users to receive tweets via simple message service (SMS) text messages on their mobile phones at no cost to the sender.

Twitter alerts do have disadvantages. The main disadvantage is a limited ability for the recipient to customize 
his or her alert criteria. The USGS Earthquake Notification System (ENS) allows users to select customized magnitude ranges and regions for their e-mail alerts [Wald et al. 2008]. Such detailed alert criteria are not possible with Twitter because messages are broadcast from accounts with multiple followers and cannot be tailored to an individual user. Controlling updates to an earthquake's hypocentral parameters and magnitude is also difficult when using Twitter. Individual users often choose to rebroadcast (retweet) older information, so the original distributor cannot control whether everyone has up-to-date information.

Several seismic networks and agencies are currently distributing Twitter earthquake alerts, including the EuropeanMediterranean Seismological Centre (EMSC) (@LastQuake), Natural Resources Canada (@CANADAquakes), and the Indonesian Meteorological Agency (@infogempabmg). The USGS issues alerts from two Twitter accounts @USGSBigQuakes and @USGSted.The @USGSBigQuakes (USGS Big Quakes) account broadcasts solutions for all earthquakes with magnitude 5.5 and larger, and includes links to supplemental products and information that are routinely produced by the USGS . The @USGSted (USGS Twitter Earthquake Dispatch) account is an experimental account that tweets alerts that include the seismological parameters along with the temporally and spatially correlated tweets-per-minute, following a seismically verified earthquake. Distributing information via multiple Twitter accounts allows followers to choose the data stream that best fits their interests.

\section{Situational awareness}

Due to the speed of tweet creation and ease of near realtime access, several groups have looked into using Twitter for gaining situational awareness following disasters. The 140character limit on tweets is both a blessing and a curse for obtaining situational awareness. The forced restriction to short messages and high user base promotes rapid dissemination and ease of content collection from potentially millions of users. The main downside is the difficulty in providing detail and actionable information in 140 characters. This restriction results in numerous tweets of marginal information value.

MacEachren et al. [2011] found success in sorting through the vast quantity of tweets and extracting potentially useful information through the implementation of a web-enabled mapping tool that collates, and plots tweets. To improve both the content and ease of automated processing, Starbird and Stamberger [2010] introduce a tweet syntax that includes parsable keywords that allow computer extraction of details such as location and content type (e.g., a victim's needs and damage descriptions). While the syntax has not been widely adopted by the content originators, volunteer efforts have arisen after disasters to translate tweets into this more actionable form [Starbird and Palen 2011].
Focusing on gathering information in the immediate aftermath of an earthquake Guy et. al. [2010] developed a prototype system that collects, collates, and analyzes tweets. The system continuously downloads tweets containing the key words "earthquake" and its equivalent in other languages. Following seismically verified earthquakes, the system produced a series of products. The products included interactive maps that show the location and text of the tweets and e-mails that contain the seismically verified location and magnitude of the earthquake, a list of the cities where the tweets were originating, and the text of the first 50 tweets after the event. They conclude that the main advantage of Twitter is speed. The qualitative descriptions contained in the tweets are available at the same time as the seismically derived earthquake parameters and sometimes provide a responding seismologist with a quick indication of the severity of the earthquake effects. The main shortcoming of Twitter-derived information is the lack of quantitative shaking estimates, such as those produced by the USGS "Did You Feel It?" and ShakeMap systems [Wald et al. 1999a, 1999b].

\section{Event detection}

The observed rapid and voluminous influx of tweets arriving shortly after earthquakes prompted several blog postings [e.g., Scoble 2008, O’Brien 2008 and O'Neill 2009] and articles [e.g., Earle et al. 2010, Li and Rao 2010, Sakaki et al. 2010] that propose using Twitter as a tool for earthquake detection. Sakaki et. al. [2010] deployed a functional system that reportedly detected and located earthquakes at speeds comparable to the Japan Meteorological Agency. Their algorithm used natural language processing to detect events and spatial particle filters to locate them. Their system operated in Japan and posted results on the web.

In this paper, we describe a simple algorithm to detect earthquakes in populated regions worldwide. We present and test an algorithm to automatically detect earthquakes using only tweets and discuss its performance, the speed of detection, and the possible use of these detections during real-time earthquake response.

\section{Implementation of an earthquake detector}

We implement a simple event detector that triggers off a rapid increase in the frequency of tweets containing the word "earthquake" or its equivalent in other languages. Rapid increases in earthquake tweets are detected using a shortterm-average over long-term-average (STA/LTA) algorithm that is commonly used in seismology to detect and time seismic phases. The algorithm requires higher-signal levels at higher-noise levels to trigger a detection, thus suppressing false triggers arising from high levels of background "noise" following highly-tweeted events and earthquake chatter unrelated to seismic events. The algorithm is based on the seismic phase picker of Earle and Shearer [1994] with a 
modification to account for the very low background frequency of earthquake tweets that often exists.

\section{Data}

Our test data consist of downloaded tweets containing the words "earthquake", "gempa", "temblor", "terremoto", or "sismo" that were collected during the time a prototype archiving system was in operation, August, 2009 through to the end of November, 2009 [Guy et. al. 2010]. Our data is limited to this timespan because the Twitter search mechanism is restricted in time and number so, unless tweets are continually downloaded, one cannot construct a continuous or complete dataset.

For each keyword-filtered tweet we archive the tweet creation time, text, and the Twitter user location. Additionally, for tweets that do not contain GPS locations, the latitude and longitude is derived from the static location string found in a user's profile using the Google Maps API Geocoding Service and stored with the tweet.

Inaccurate tweet geo-locations are a serious issue when using geospatially related tweets. The vast majority of tweets do not contain GPS locations, and the tweet location is often only as accurate as the static location string the user entered in their Twitter profile. A location is not required when setting up a Twitter account and can be as vague or specific as the user wants. Given this, a tweet from a New Yorker on vacation in San Francisco will mis-locate to New York. These types of location errors are troublesome but are not spatially correlated, so averaging can minimize their negative effects.

Our goal is to identify tweets originating from users who experienced earthquake shaking and not increases in twitter activity following the release of news or blog articles. To minimize such contamination, we remove all tweets that contain Uniform Resource Locators (URLs), specifically the string "http". Furthermore, we remove all tweets with the text "RT" or "@”." "RT" is commonly used to identify a tweet as a rebroadcast or "retweet" of another users tweet and the "@" symbol is used in front of a username to reply to another user's tweet. Thus tweets containing "@” and "RT" are likely to arise from a user commenting on an earthquake from outside the felt area.

\section{Detection algorithm}

Figure 1 illustrates the detection algorithm. Using our culled dataset we generate a tweet-frequency time series or 'tweetgram' by binning tweets into five-second windows and normalizing to tweets-per-minute (Figure 1A). A characteristic function (Figure 1B) is generated from the tweetgram and is used to declare event detections. The characteristic function is defined as:

$$
\mathrm{C}(t)=\mathrm{STA} /(m \mathrm{LTA}+b)
$$

The characteristic value $C(t)$ is calculated for every time bin and a detection is declared when $C(t)$ exceeds 1 . The STA is the short-term average taken one minute before time $t$ and LTA is the long-term average taken 60 minutes before the STA. The constants $m$ and $b$ are tunable parameters that define the sensitivity of the detection. The LTA approximates the 'background noise' and the STA approximates the tweets

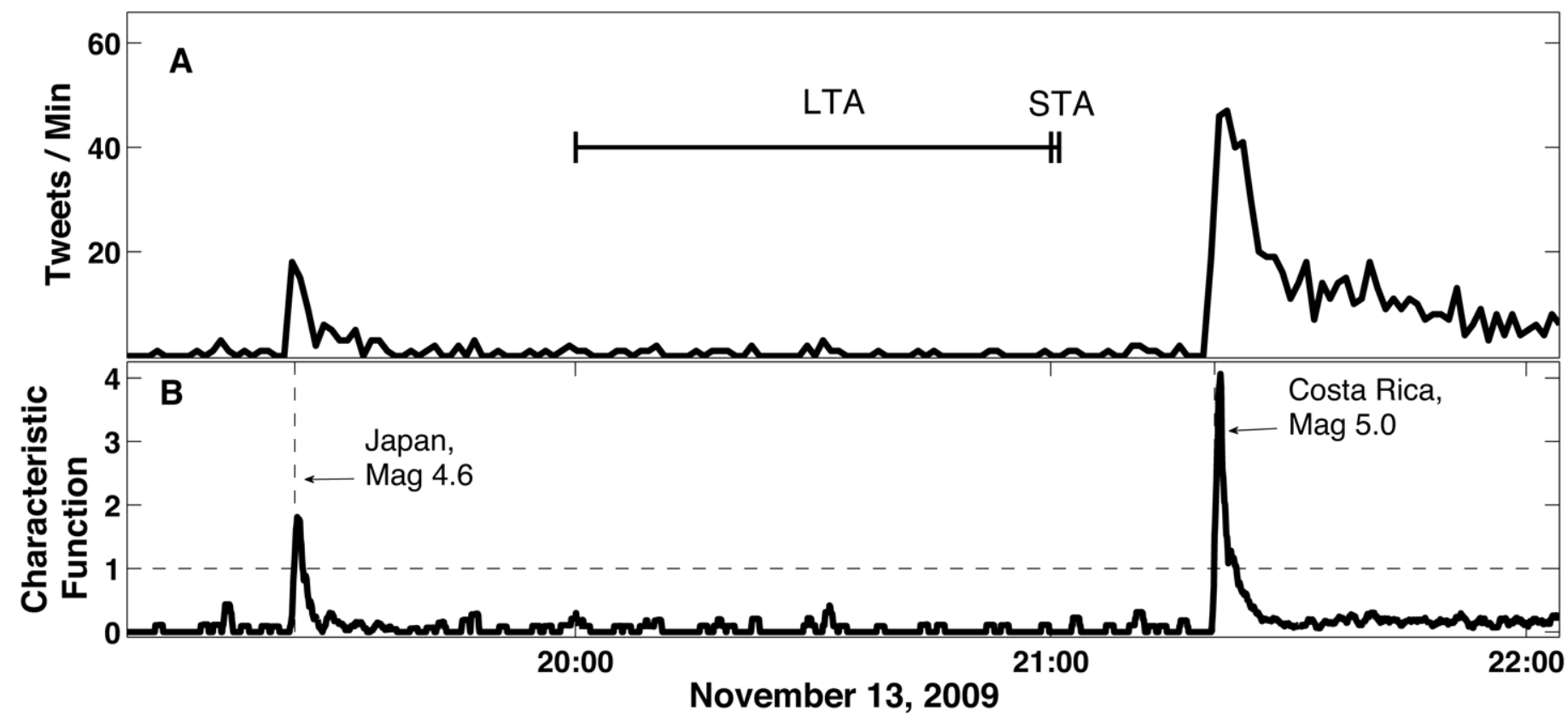

Figure 1. Illustration of the detection process (see text for details). A) Tweet-frequency or tweetgram for about three hours of tweets on November 13, 2009, in UTC. B) The characteristic function generated from the tweetgram in (A). The characteristic function has a one minute STA, a sixty minute LTA, $m=4$, and $b=10$. 


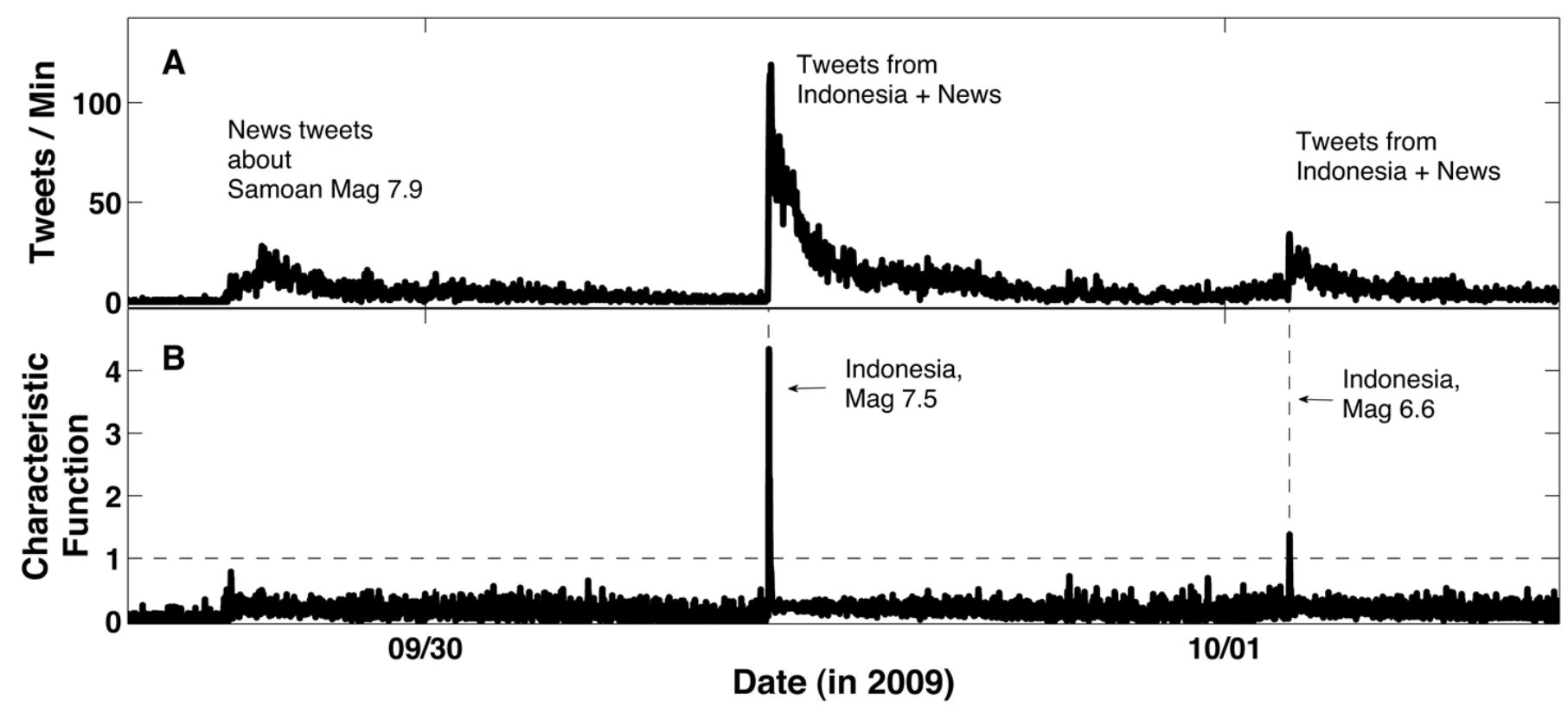

Figure 2. Several days of tweets spanning three events in September and October 2009. Our detector is tuned for sudden increases in Twitter activity, common for tweets submitted after felt shaking. When increases in Twitter activity results from news reports, as in the Samoan event, the tweet frequency increases more gradually and the characteristic function sometimes does not trigger. This is the preferred behavior because we are interested in tweets resulting from felt events.

in response to a possible event or 'signal' plus the background noise. $C(t)$ requires higher signal levels (STA) to trigger at higher noise levels (LTA). The constant $b$ has units of tweetsper-minute and defines the STA value that will cause an event trigger when the LTA is zero. The constant $m$ defines the increased STA necessary to trigger at a larger LTA. We require $\mathrm{C}(t)$ to drop to 0.25 before another trigger is allowed to occur. This requirement limits multiple triggers that can be generated for the same event due to a $\mathrm{C}(t)$ that oscillates around one; however, it also limits our ability to detect very closely spaced events.

The detector has proven effective at differentiating between sudden, sharp increases in twitter activity emanating from people who felt an earthquake from more gradual increases that result as news of an event propagates around the world. Figure 2 shows one such example. For two events in Indonesia, tweets reporting the earthquake rapidly came from within the expected felt area. Within two minutes of these earthquakes, our detection algorithm correctly identified the events. In the case of the Samoa earthquake, Twitter activity was mainly from referencing news reports and thus shows a more gradual, emergent development. We collected no immediate tweets from Samoa communities and the detector did not trigger on this event. Triggering on an event that is already known and announced is not the goal of this detector.

The STA/LTA approach also helps mitigate the effect of variations in background noise due to time of day and unrelated world events. We need to be able to detect spikes above the background chatter that might occur from "earthquake" tweets unrelated to actual earthquakes. For example, one can envision a higher level of "earthquake" tweets the day a Dairy Queen has a two-for-one offer on their "Oreo earthquake brownie" ice cream sundae. Additionally, we want to avoid false triggers during the times of high activity following earlier significant earthquakes.

\section{Detector performance}

We tested the performance of the detector on our tweet data that spanned that spanned August, 2009 through the end of November, 2009. As with most automatic detection methods, there is a trade-off between the number of false triggers and the number of missed events. We tuned our algorithm by: 1) generating a large set of triggers using low values of $m$ and $b, 2$ ) identifying each trigger as 'verified', 'false', or 'possible' and 3) performing a four-dimensional grid search over LTA, STA, $b$ and $m$ to find parameters that produce a high ratio of verified to false triggers.

An event is considered 'verified' if an earthquake exists in a global or regional seismic catalog that plausibly could have been felt in the area where the majority of the tweets originated and if the text of the tweets are qualitatively consistent with the response to shaking. The absence of an earthquake from a seismic catalog does not mean it did not occur; earthquake often go undetected in areas where seismic instrumentation is sparse. Therefore, our Twitter detector may find earthquakes that are not reported in seismic catalogs. To account for this possibility, we identify a trigger as a possible quake if the tweet text is consistent with that of verified events and the tweets are clustered in space and time. A trigger is categorized as 'false' if the tweet text is clearly unrelated to a shaking event, such as an 


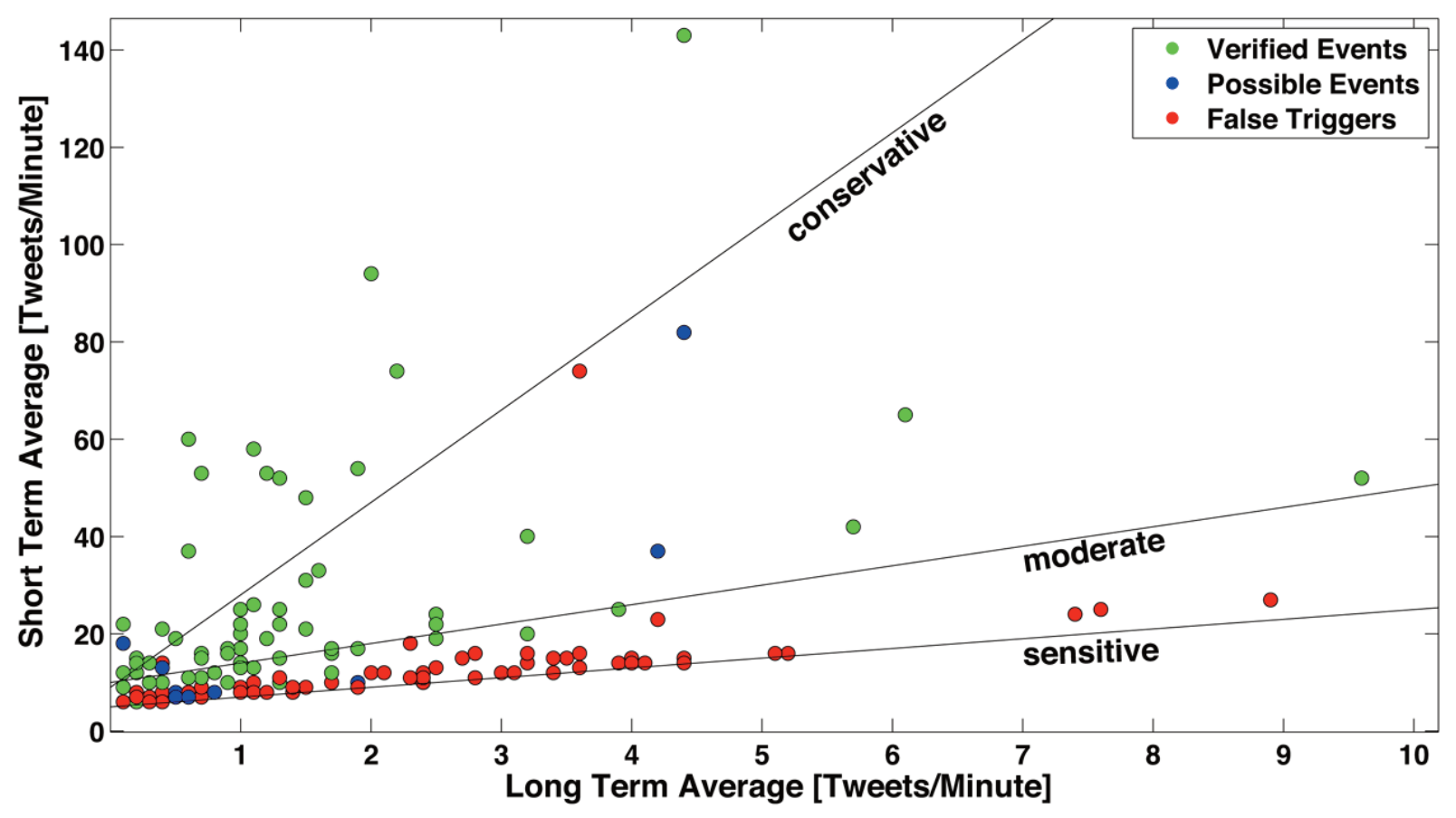

Figure 3. Recorded STA versus LTA for events in our dataset. Green dots, 'verified' earthquakes; blue dots, 'possible' earthquakes; red dots, 'false' triggers (see text for details). Note that these STA and LTA values correspond to where the characteristic function reaches its highest value following a trigger. The three lines are defined as STA $=m^{*}$ LTA $+b$ and represent the three trigging thresholds discussed in the text. Events above a given line will trigger a detection for the corresponding trigger sensitivity. More conservative thresholds produce a higher ratio of verified to false triggers.

earthquake drill or chatter about an epic session of the Quake video game.

Figure 3 summarizes the results of our test. For each event trigger, the plot shows the STA and LTA values for the event's highest value of the characteristic function following the trigger. Seismically verified earthquakes are plotted as green circles, possible events as blue circles, and nonearthquake events as red circles. The lines are defined as STA $=m^{*} \mathrm{LTA}+b$. The lines correspond to three different triggering thresholds: sensitive $(m=2$ and $b=5)$, moderate ( $m=4$ and $b=10$ ), and conservative trigger $(m=19$ and $b=9$ ). Points plotted above a given line will generate a detection for a $C(t)$ with the corresponding values of $m$ and $b$.

Table 1 summarizes the number of 'verified', 'possible' and 'false' triggers for these threshold values. The 'moderate' sensitivity trigger provides a good trade-off between catching major events and producing a low number of false triggers. A better assessment of the detector performance can be obtained if we tune and test the detector on independent datasets. This procedure would require a longer time series than we currently have and it is left for future work. In Figure 3, note that events trigger as soon as $C(t)$ exceeds 1 and the points shown represents the maximum value reached by the characteristic function following the detection of that event.

For false triggers, it is often clear from reading the tweet text that they are not associated with an earthquake. The only two false triggers caught for the 'moderate' sensitivity trigger resulted from tweets during the Great California ShakeOut earthquake drill (www.shakeout.org) on October

\begin{tabular}{lccc}
\hline Trigger & $\begin{array}{c}\text { Verified } \\
\text { earthquakes }\end{array}$ & $\begin{array}{c}\text { Possible } \\
\text { earthquakes }\end{array}$ & $\begin{array}{c}\text { False } \\
\text { triggers }\end{array}$ \\
\hline Sensitive & 61 & 10 & 75 \\
Moderate & 48 & 3 & 2 \\
Conservative & 18 & 1 & 0 \\
\hline
\end{tabular}

Table 1. Number of verified, possible, and false triggers for different trigger sensitivities. Detection time is very fast, with about $75 \%$ coming in before two minutes.

15, 2009 and the space shuttle landing on September 12, 2009. In the case of the space-shuttle landing, many of the tweets reported something to the effect of, "That was not an earthquake it was the space-shuttle sonic boom". In fact, shortly before the shuttle's sonic boom a Twitter user tweeted "... that loud noise you're about to hear is not an earthquake. Sonic Boom”. While it is easy for a human to understand and interpret tweet text, getting a computer to interpret natural language is difficult. This is especially true in Twitter, where we see multiple languages and countless abbreviations. Such computerized language interpretation is pursued by [e.g., Sakaki et al. 2010 and Palen et al. 2010b].

Figure 4 maps the verified, potential, and false detections for the moderate sensitivity trigger. Verified events are plotted at the location of the corresponding cataloged earthquake, and potential and false triggers are plotted at the location of the highest tweet density. The map shows 


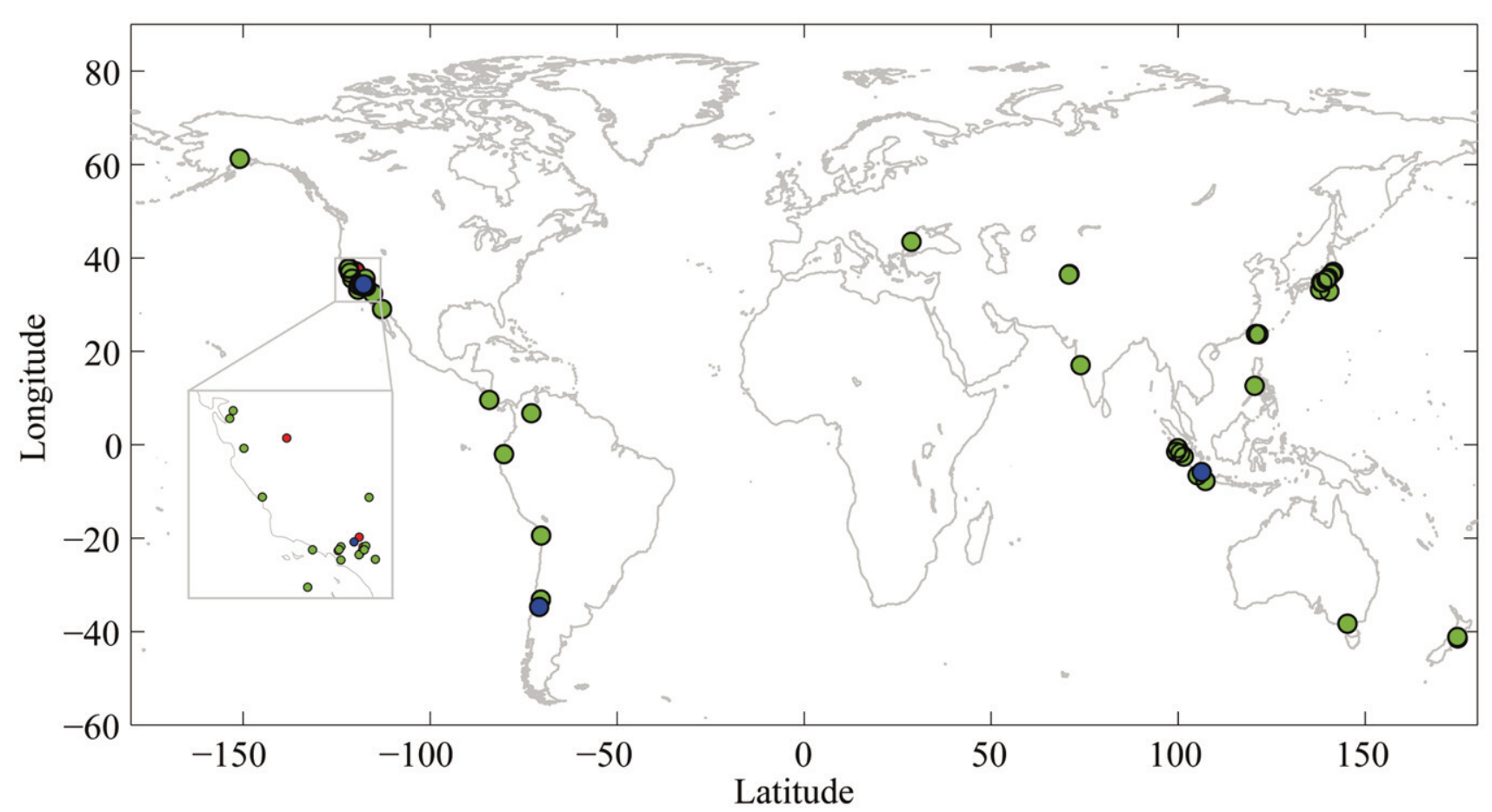

Figure 4. Map showing events caught by our detector using the 'moderate' threshold. Green dots, 'verified' earthquakes; blue dots, 'possible' earthquakes; red dots, 'false' triggers. The inset map shows an expanded view of California where the two false triggers occurred.

reasonable global coverage with the highest number of detections in California, Japan, and Indonesia. These results show the potential global reach of the method, with some detections occurring in sparsely instrumented regions where seismically obtained detections can be slow, or small felt earthquakes could potentially be missed.

The detection time is not the origin time of the earthquake or the initiation of the tweets, both of which will precede the detection time because it takes time for the STA value to sufficiently increase to cause a trigger. Also, for off-shore events or events with epicenters outside of populated regions, it can take time for the shaking to reach Twitter users. The detection times also depend on the characteristics of the tweetgram. Slower increases in tweets-per-minute will result in delayed triggers, if these trigger at all. Figure 5 shows a histogram of the detection time for verified events found using the moderate sensitivity trigger. Despite the mentioned delays, the detection time for events is very fast; roughly $75 \%$ of events trigger within two minutes. In most cases, this precedes

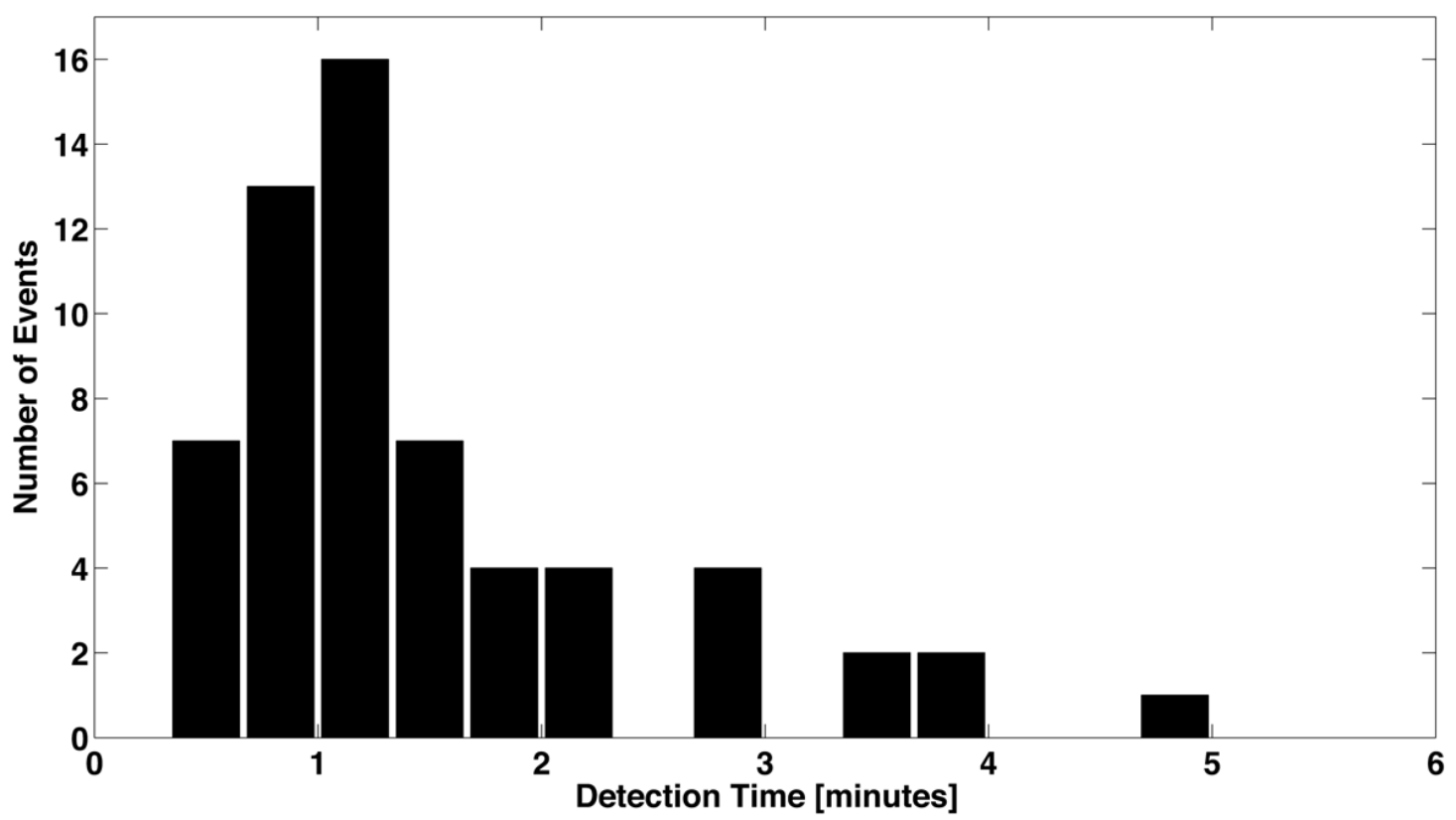

Figure 5. Detection time for earthquakes found using the 'moderate' sensitivity threshold. Detection time is very fast, with about $75 \%$ coming in before two minutes. 
the USGS public release of seismically derived hypocenters and magnitudes that range from two minutes in wellinstrumented regions to 20 minutes for sparsely instrumented regions of the world.

\section{Discussion}

We have demonstrated that earthquake detections based solely on Twitter messages are possible for earthquakes worldwide with a low rate of false triggers. Now we briefly explore their potential use.

Our twitter-based earthquake detector misses the vast majority of seismically detected events. For comparison, the USGS global earthquake catalog (http: / / earthquake.usgs.gov / research/data/pde.php) contains 5,175 earthquakes while only 48 were found by the Twitter detector with a moderate threshold. The majority of seismically detected earthquakes are either too small to produce perceivable shaking or occur outside populated areas. Twitter use is also far from ubiquitous and not all Twitter users who feel an earthquake report it. The detections, however, are generally caused by widely felt events that are of more immediate interest than those with no human impact. These detected events occurred near urban areas and range in magnitude from 2.0 to 7.5 with a median magnitude of 4.6 .

Given this high percentage of missed events and the inability to extract the accurate hypocentral and magnitude estimates that are needed for impact assessment, Twitter is in no way a replacement for a seismic network. The potential benefits from Twitter arise from Twitter's significant global penetration, the rapid submission and access to tweets, and the short narratives contained in the tweets. Possible uses include, discovery of earthquakes in sparsely instrumented regions, earthquake detection before seismically derived parameters are obtained, and situational awareness provided by the text of the tweets.

The USGS National Earthquake Information Center (NEIC) strives for a complete earthquake catalog at a magnitude threshold of 4.5 globally and 2.5 in wellinstrumented areas of the USA. Additionally, NEIC reports on smaller earthquakes globally, if they are widely felt and/or news worthy. The vast majority of these earthquakes are detected by the roughly 2,000 seismic stations that flow into NEIC's real-time location system. However, occasionally events meeting these criteria, in poorly instrumented regions, are missed. These missed events are sometimes first noticed by citizen reports submitted to the USGS "Did You Feel It?" system or phone calls to the NEIC. If an approximate time and location of an earthquake that was missed by our automatic system is known, it is sometimes possible for a seismologist to manually extract a location and magnitude. Twitter offers another mechanism to discover these small events. Although the number of earthquakes with "Did You Feel It?" responses exceeds the 48 with Twitter reports, 11 of the 48 events we detected using Twitter during our five month test have no corresponding "Did You Feel It?" responses.

Twitter-based detections have the ability to rapidly alert seismic monitoring agencies that a widely felt earthquake occurred, potentially before seismic systems have detected the earthquake. The majority of our Twitter detections occur in less than two minutes (Figure 5). This is faster than many earthquakes are detected by NEIC's automatic systems. For example, The NEIC first seismically detected the recent magnitude 9.0 earthquake in Japan 3.8 minutes after it occurred [Hayes et al. 2011]. The initial NEIC detection had a location but no magnitude. At this same time, "earthquake" tweets spanned the entire island of Honshu and southern Hokkaido. Such a rapid and geographically widespread Twitter response is a good indication that an event was widely felt and potentially required the mobilization of additional USGS response personnel. In the case of Japan, improved response time can be obtained from increased sharing of seismological waveform and parametric data. This effort is underway, but most regions of the world are not as well instrumented as Japan.

The focus of this discussion has been on using Twitter for augmenting instrumentally derived data. Other socialnetworking tools and Internet data-mining techniques have similar potential. Automatically scanning other services such as Facebook and Flickr could yield firsthand accounts and damage photos. Internet-based systems that do not rely on social networking are also feasible. The EuropeanMediterranean Seismological Centre (EMSC) has implemented a system to map out the felt region of an earthquake by geocoding the IP addresses of people visiting its website [Bossu et al. 2008]. The rapid increase in website visits is often EMSC's first indication that an earthquake has occurred. Their system has proven so robust that initial indications of felt earthquakes derived from nonseismological data are publically posted prior to the seismologically derived parameters.

This and previous studies show the potential for Twitter and other social-media tools to aid in earthquake response. These tools are, at their root, a way for people to communicate, and millions of people are using them. After natural disasters people have used, and will continue to use, social media to describe what they saw and experienced.

Scientifically derived products such as shaking estimates from ShakeMap and impact estimates from the USGS Prompt Assessment of Global Earthquakes for Response (PAGER) system [Wald et al. 2010] ,once derived, replace the majority of the information that can be obtained from Twitter. The basic benefit from Twitter-based information is speed and the low cost of accessing and analyzing the data. The challenge is how best to summarize and present the often content-limited messages. 


\section{References}

Bossu, R., G. Mazet-Roux, V. Douet, S. Rives, S. Marin and M. Aupetit (2008). Internet users as seismic sensors for improved earthquake response, Eos Trans. AGU, 89, 225-226.

Earle, P. and P. Shearer (1994). Characterization of global seismograms using an automatic-picking algorithm, B. Seismol. Soc. Am., 84, 366-376.

Earle, P., M. Guy, R. Buckmaster, C. Ostrum, S. Horvath and A. Vaughan (2010). OMG earthquake! Can Twitter improve earthquake response?, Seismol. Res. Lett., 81, 246-251.

Guy, M., P. Earle, C. Ostrum, K. Gruchalla and S. Horvath (2010). Integration and dissemination of citizen reported and seismically derived earthquake information via social network technologies, Lect. Notes Comput. Sci., 6065, 42-53.

Hayes, G.P., P.S. Earle, H.M. Benz, D.J. Wald, R.W. Briggs and the USGS/NEIC Earthquake Response Team (2011). Eighty-eight hours: the U.S. Geological Survey National Earthquake Information Center response to the 11 March 2011 Mw 9.0 Tohoku earthquake, Seismol. Res. Lett., 82, 481-493; doi: 10.1785 / gssrl.82.4.481.

Li, J. and H.R. Rao (2010). Twitter as a rapid response news service: An exploration in the context of the 2008 China earthquake, Electr. J. Inform. Syst. Develop. Countries, 42, 1-22.

Liu, S.B. and A. Ayala Iacucci (2010). Crisis map mash-ups in a participatory age, American Congress on Surveying and Mapping (ACSM) Bulletin, June 2010, 10-14.

MacEachren, A.M., A.C. Robinson, A. Jaiswal, S. Pezanowski, A. Savelyev, J. Blanford and P. Mitra (2011). Geo-Twitter analytics: applications in crisis management, Proceedings, 25th International Cartographic Conference, Paris, France.

Mendoza, M., B. Poblete and C. Castillo (2010). Twitter under crisis: can we trust what we RT?, In: Proceedings of the First Workshop on Social Media Analytics, ACM, New York, 71-79.

O’Brien, C. (2008). Is Twitter the Newsroom of the Future?; http: / / www.pbs.org/idealab / 2008 / 07 / is-twitter-thenewsroom-of-the-future005.html.

O’Neill, I. (2009). The anatomy of a Los Angeles earthquake... on Twitter; http: / /www.astroengine.com/2009/ 01 / the-anatomy-of-a-los-angeles-earthquake-on-twitter/).

Palen, L., K. Starbird, S. Vieweg and A. Hughes (2010a). Twitter-based information distribution during the 2009 Red River Valley flood threat, B.. Am. Soc. Inform. Sci. Technol., 36, 13-17.

Palen, L., K.M. Anderson, G. Mark, J. Martin, D. Sicker, M. Palmer and D. Grunwald (2010b). A vision for technology-mediated support for public participation and assistance in mass emergencies and disasters, In: Proceedings of the 2010 ACM-BCS Visions of Computer Science Con- ference (Edinburgh, United Kingdom, April 14 - 16, 2010). ACM-BCS Visions of Computer Science, British Computer Society, Swinton, UK, 1-12.

Sakaki, T., M. Okazaki and Y. Matsuo (2010). Earthquake shakes Twitter users: real-time event detection by social sensors, In: Proceedings of the 19th International Conference on World Wide Web, ACM, New York, 851-860.

Scoble, R. (2008). Twittering the earthquake in China; http: / / scobleizer.com/2008/05/12/ quake-in-china/.

Starbird, K. and J. Stamberger (2010). Tweak the tweet: leveraging microblogging proliferation with a prescriptive sytax to support citizen reporting, In: Proceedings of the 7th International ISCRAM Conference, Seattle.

Starbird, K. and L. Palen (2011). 'Voluntweeters': self organizing by digital volunteers in times of crisis, In: Proceedings of the 2011 Annual Conference on Human Factors in Computing Systems, Vancouver, Canada.

Wald, L.A., D.J. Wald, S. Schwarz, B. Presgrave, P.S. Earle, E. Martinez and D. Oppenheimer (2008). The USGS Earthquake Notification Service (ENS): customizable notifications of earthquakes around the globe, Seismol. Res. Lett., 79 (1), 103-110.

Wald, D.J., V. Quitoriano, L. Dengler and J.W. Dewey (1999a). Utilization of the Internet for Rapid Community Intensity Mapss, Seismol. Res. Lett., 70, 680-697.

Wald, D.J., V. Quitoriano, T.H. Heaton, H. Kanamori, C.W. Scrivner and B.C. Worden (1999b). TriNet "ShakeMaps" - rapid generation of peak ground-motion and intensity maps for earthquakes in southern California, Earthqu. Spectra 15, 537-556.

Wald, D.J., K.S. Jaiswal, K.D. Marano, D.B. Bausch and M.G. Hearne (2010). PAGER — Rapid assessment of an earthquake's impact, U.S. Geological Survey Fact Sheet 20103036, 4 p.

\footnotetext{
${ }^{\star}$ Corresponding author: Paul S. Earle,

U.S. Geological Survey, Denver, CO, USA; email: pearle@usgs.gov.

C 2011 by the Istituto Nazionale di Geofisica e Vulcanologia. All rights reserved.
} 